\title{
Efecto de la quercetina sobre la tasa de desarrollo y la viabilidad de embriones bovinos producidos in vitro
}

\author{
Effect of quercetin on the rate of development and viability of bovine embryos \\ produced in vitro
}

\author{
Diana Maturana ${ }^{1,3}$, Jorge Gómez O. ${ }^{1}$, Giovanni Restrepo B. ${ }^{2}$
}

\section{Resumen}

\begin{abstract}
Las tasas de desarrollo y la calidad de los embriones bovinos producidos in vitro son menores que los producidos in vivo, debido al estrés oxidativo al que son sometidos durante su manipulación y las condiciones de cultivo. El objetivo de este estudio fue evaluar la producción, la celularidad y la vitalidad de los blastocistos bovinos producidos in vitro con diferentes concentraciones de quercetina. Un total de 2108 oocitos fueron madurados in vitro en medio 199 con $10 \%$ de SFB, $5.0 \mu \mathrm{g} / \mathrm{ml}$ de LH y $0.5 \mu \mathrm{g} / \mathrm{ml} \mathrm{de}$ FSH. La fertilización se realizó en medio TALP con $2 \times 10^{6}$ espermatozoides $/ \mathrm{ml}$. Los posibles cigotos fueron cultivados a $38.7{ }^{\circ} \mathrm{C}$ con $5 \%$ de $\mathrm{CO}_{2}$ en medio de cultivo SOF suplementado con seis concentraciones de quercetina $(1,5,10,15,20,50 \mu \mathrm{M})$ y como controles se utilizaron el medio SOF sin quercetina y suplementado con DMSO. Se determinaron las tasas de clivaje (día 2) y blastocistos (día 7), así como la celularidad y la viabilidad celular de los blastocistos. Se realizó un análisis de varianza y las medias de los tratamientos se compararon con la prueba de Tukey. Los tratamientos con 1 y $5 \mu \mathrm{M}$ de quercetina fueron superiores para la tasa de blastocistos $(\mathrm{p}<0.05)$. Se observó una reducción en la tasa de clivaje, la tasa de blastocistos y la viabilidad celular para 20 y $50 \mu \mathrm{M}$, y se encontró una disminución en la celularidad para $15 \mu \mathrm{M}$. La quercetina utilizada a bajas concentraciones durante el cultivo in vitro de embriones bovinos produce un incremento en la tasa de blastocistos, mientras que el uso altas concentraciones de quercetina genera efectos deletéreos en los embriones bovinos.
\end{abstract}

Palabras clave: antioxidante; estrés oxidativo; fertilización in vitro; radical libre

\footnotetext{
${ }^{1}$ Grupo de Investigación en Biotecnología Animal (GIBA), Facultad de Ciencias Agrarias, Politécnico Colombiano Jaime Isaza Cadavid, Medellín, Colombia

${ }^{2}$ Grupo de Investigación en Biotecnología Animal (GIBA), Facultad de Ciencias Agrarias, Universidad Nacional de Colombia, Medellín, Colombia

${ }^{3}$ E-mail:dmmatura@unal.edu.co
}

Recibido: 9 de noviembre de 2018

Aceptado para publicación: 5 de mayo de 2019 
The development rates and the quality of the bovine embryos produced in vitro are lower than those produced in vivo, due to the oxidative stress to which they are subjected during their manipulation and the culture conditions. The aim of this study was to evaluate the production, cellularity and vitality of bovine blastocysts produced in vitro with different concentrations of quercetin. A total of 2108 oocytes were matured in vitro in medium 199 with $10 \% \mathrm{FBS}, 5.0 \mu \mathrm{g} / \mathrm{ml} \mathrm{LH}$ and $0.5 \mu \mathrm{g} / \mathrm{ml} \mathrm{FSH}$. Fertilization was carried out in TALP medium with $2 \times 10^{6}$ spermatozoa $/ \mathrm{ml}$. Potential zygotes were cultured at $38.7^{\circ} \mathrm{C}$ with $5 \% \mathrm{CO}_{2}$ in SOF culture medium supplemented with six concentrations of quercetin $(1,5,10,15,20,50 \mu \mathrm{M})$ and as controls were used the SOF medium without quercetin and supplemented with DMSO. The rates of cleavage (day 2) and blastocysts (day 7) were determined, as well as the cellularity and cell viability of the blastocysts. An analysis of variance was performed, and the means of the treatments were compared with the Tukey test. Treatments with 1 and $5 \mu \mathrm{M}$ of quercetin were higher for the blastocyst rate $(\mathrm{p}<0.05)$. A reduction in the cleavage rate, the blastocyst rate and cell viability were observed for 20 and $50 \mu \mathrm{M}$, and a decrease in cellularity for $15 \mu \mathrm{M}$ was found. Quercetin used at low concentrations during the in vitro culture of bovine embryos produces an increase in the rate of blastocysts, while the use of high concentrations of quercetin generates deleterious effects in bovine embryos.

Key words: antioxidant; oxidative stress; in vitro fertilization; free radical

\section{INTRODUCCIÓN}

La producción de embriones in vitro ha permitido numerosos avances en el mejoramiento genético de los bovinos, y ha sido ampliamente utilizada a nivel mundial con fines investigativos y comerciales. No obstante, entre el 50 y el $70 \%$ de los posibles cigotos detienen su desarrollo a los 5-7 días del cultivo in vitro (Zullo et al., 2016). Las bajas tasas de desarrollo embrionario in vitro están asociadas a las subóptimas condiciones de los medios de cultivo, la manipulación excesiva, la concentración de oxígeno y la alta exposición a la luz, que generan un incremento en la producción de especies reactivas de oxígeno (EROs) (Olson y Seidel, 2000; Machado et al., 2011; Merton et al., 2013).

El exceso de EROs conlleva a una condición de estrés oxidativo que puede conducir a daños en el ADN y a la peroxidación de los lípidos, lo cual afecta la viabilidad celular, y podría inducir la muerte programada de las células (Guérin et al., 2001). Por lo tanto, el desbalance de EROs en los cultivos de embriones in vitro está altamente relacionado con la baja la calidad de los blastocistos producidos, que a su vez está directamente relacionado a los bajos porcentajes de preñez (Kuwayama y Leibo, 2008; Caamaño et al., 2015; Zullo et al., 2016).

La adición de moléculas antioxidantes en las diferentes etapas de la producción de embriones, han mostrado un incremento en las tasas de blastocistos; entre estas, la carnitina, el resveratrol y la quercetina (Rahim et al., 2011; Guemra et al., 2013; Salzano et al., 2014; Kang et al., 2016; Truong y Gardner, 2017).

La carnitina es un antioxidante que se ha suplementado durante la maduración in vitro (MIV) de los ovocitos bovinos, mejorando la maduración nuclear y desarrollo de los embriones, pero la molécula no presenta 
ningún efecto benéfico cuando los embriones son vitrificados (Phongnimitr et al., 2013). Por otro lado, el resveratrol es un flavonoide que aumenta las tasas de blastocistos cuando se suplementa durante la maduración in vitro de oocitos bovinos (Wang et al., 2014; Torres et al., 2016; Kordowitzki et al., 2016; Sovernigo et al., 2017), porcinos (Kwak et al., 2012) y caprinos (Mukherjee et al., 2014). Además, aumenta el desarrollo embrionario durante el cultivo in vitro de embriones (Lee et al., 2010) y la criotolerancia de los blastocistos (Giaretta et al., 2013). Así mismo, Salzano et al. (2014) encontraron que la concentración de $0.5 \mu \mathrm{M}$ aportó criotolerancia a los embriones, obteniendo mejores resultados en la sobrevivencia a la vitrificación. Sin embargo, las aplicaciones del uso del resveratrol podrían estar limitadas, ya que los precursores que se emplean para su obtención son costosos para aplicaciones industriales (Li et al., 2015), de allí la importancia de evaluar otras moléculas que puedan tener efectos similares o superiores al resveratrol a un menor costo.

Otro flavonoide perteneciente al grupo de los polifenoles que ha sido estudiado es la quercetina. Se han demostrado su eficacia para atrapar radicales libres en cultivos celulares, inclusive siendo superior a los antioxidantes tradicionales como las vitaminas C y E (Gordon y Roedig-Penman, 1998; Ishige et al., 2001; Geetha et al., 2005; Liu et al., 2010), y se le encuentra fácilmente en la naturaleza (Wang et al., 2016). Se han reportado resultados positivos sobre las tasas de maduración y producción de blastocistos al suplementar la quercetina a bajas concentraciones en el medio de maduración en diferentes especies; así, Banihosseini et al. (2018) la utilizaron en concentración de 16 y $30 \mu \mathrm{M}$, mejorando la maduración y el desarrollo de embriones murinos. Adicionalmente, Sovernigo et al. (2017) informaron que no hubo diferencia respecto a las tasas de clivaje de oocitos bovinos madurados en medios suplementados con quercetina, vitamina $\mathrm{C}$, cisteamina, carnitina y resveratrol, ni para la eclosión de los blastocistos.
Se dispone de escasos reportes sobre el efecto de la quercetina en la producción in vitro de embriones bovinos. Sin embargo, se conoce que en la fase de maduración in vitro permite un incremento en la tasa de producción de blastocistos, al ser utilizada en concentraciones entre 2 y $10 \mu \mathrm{M}$ (Guemra et al., 2013). El objetivo de esta investigación fue evaluar el efecto de la quercetina sobre la tasa de desarrollo y la viabilidad de embriones bovinos producidos in vitro.

\section{Materiales y Métodos}

\section{Obtención de Oocitos}

Se recolectaron ovarios de hembras bovinas faenadas en el frigorífico de la Central Ganadera S.A de Medellín, Colombia, y transportados durante 30 minutos en solución salina estéril al $0.9 \%$ a $35^{\circ} \mathrm{C}$ hasta el laboratorio del Politécnico Colombiano Jaime Isaza Cadavid, en la sede de Bello, Antioquía.

Los complejos cúmulus oocitos (COCs) fueron obtenidos mediante la aspiración de folículos con diámetros entre 2 y $6 \mathrm{~mm}$, usando una jeringa de $5 \mathrm{ml}$ y una aguja hipodérmica estéril calibre 18 (González et al., 1992; Coelho et al., 2002; Moraes et al., 2002; Calegari et al., 2012). El líquido folicular fue depositado en tubos estériles de $50 \mathrm{ml}$, mantenidos en baño maría a $35^{\circ} \mathrm{C}$ durante el proceso de aspiración. Los complejos COCs fueron recuperados mediante un filtro de $70 \mu \mathrm{m}$ (Falcon ${ }^{\mathrm{TM}}$, EEUU) y depositados en Solución Buffer Fosfato (PBS) para ser seleccionados y clasificados. Los COCs aptos para la maduración se evaluaron teniendo en cuenta tres parámetros: citoplasma homogéneo, morfología homogénea y mínimo tres capas de células de la granulosa (Leibfried y First, 1979).

\section{Maduración in vitro (MIV)}

Los CCOs fueron incubados en gotas de $70 \mu 1$ de medio de maduración (Gibco 11150-059) suplementado con $0.33 \mathrm{mM}$ de 
piruvato de sodio (Sigma, EEUU), $1 \mu \mathrm{g} / \mathrm{mL}$ de estradiol (Sigma, EEUU), 10\% de suero fetal bovino (SFB) (Sigma), $83.4 \mu \mathrm{g} / \mathrm{ml} \mathrm{de}$ amikacina (Genfar ${ }^{\circledR}$, Colombia), $50 \mathrm{UI} / \mathrm{ml}$ LH (Chorulon ${ }^{\circledR}$ ), $1 \mu \mathrm{g} / \mathrm{ml}$ de FSH (Bioniche, Canadá). Las gotas fueron cubiertas con aceite mineral (Sigma) e incubadas a $38.7{ }^{\circ} \mathrm{C}$, $5 \%$ de $\mathrm{CO}_{2}$ en aire con $90 \%$ de humedad relativa por 24 horas (Guemra et al., 2013).

\section{Fertilización in vitro}

Para la fertilización in vitro (FIV) de los COCs previamente madurados se utilizó semen criopreservado de un donante de la raza Blanco Orejinegro. El semen fue descongelado en agua a $37^{\circ} \mathrm{C}$ durante 1 minuto. Los espermatozoides móviles fueron seleccionados por un gradiente discontinuo de Percoll ${ }^{\circledR} 45 \%$ - 90\% adicionando $250 \mu 1$ en un tubo de $1.5 \mathrm{ml}$. El semen fue adicionado en la parte superior del gradiente de Percoll $\AA$ (Sigma), el cual fue sometido a centrifugación a 800 g por 5 minutos (Eppendorf ${ }^{\circledR}$, Alemania). El sobrenadante fue retirado y el pellet fue resuspendido en $400 \mu 1$ de medio FertTALP y luego centrifugado por 3 minutos a $400 \mathrm{~g}$ (Parrish, 2014).

El medio de FIV fue suplementado con $2 \mu \mathrm{M}$ de penicilamina (Sigma), $1 \mu \mathrm{M}$ de hipotaurina (Sigma), $250 \mu \mathrm{M}$ de epinefrina (Sigma), $20 \mu \mathrm{g} / \mathrm{mL}$ de heparina (Sigma) y 6 $\mathrm{mg} / \mathrm{ml}$ de albúmina sérica bovina (BSA) (Sigma). Los gametos fueron incubados durante 18 horas en gotas de $60 \mu 1$ de medio FIV cubiertas con aceite mineral (Sigma), con una concentración de $2 \times 10^{6}$ espermatozoides/ml (Guemra et al., 2013).

\section{Cultivo in vitro}

Pasadas las 18 horas de la fertilización, se retiraron las células del cúmulus por pipeteo y los posibles cigotos fueron transferidos al medio fluido sintético oviductal (SOF) (Holm et al., 1999), el cual fue suplementado con $0.33 \mathrm{mM}$ de piruvato de sodio (Sigma), $3 \%$ de aminoácidos esenciales (Sigma), 1\% de no esenciales (Sigma), $0.34 \mathrm{mM}$ de tricitrato de sodio (Sigma), $2.7 \mathrm{mM}$ de mioinositol (Sigma) (Holm et al., 1999), 5\% de SFB (Gibco, EE.UU), $5 \mathrm{mg} / \mathrm{ml}$ de BSA y quercetina (Sigma) diluida en dimetilsulfóxido (DMSO) (Sigma) a diferentes concentraciones $(1,5,10,15,20,50 \mu \mathrm{M})$. Como controles se empleó el medio SOF sin antioxidante y SOF con DMSO a una relación $1 \mu \mathrm{l} / \mathrm{ml}$, debido a que fue el solvente utilizado para diluir el antioxidante.

El cultivo se realizó en gotas de $70 \mu 1$ cubiertas de aceite mineral. El medio fue mantenido en incubadora a $38.7{ }^{\circ} \mathrm{C}$ y $5 \%$ de $\mathrm{CO}_{2}$. Pasadas 48 horas se determinó el porcentaje de división y se realizó el recambio del $50 \%$ del medio de cultivo. En el día 6 de desarrollo se realizó un último recambio del $50 \%$ del medio de cultivo y en el día 7 se determinaron las tasas de producción de embriones.

\section{Viabilidad de los Embriones}

Los blastocistos expandidos fueron transferidos durante 1 minuto a tubos de 0.6 $\mathrm{ml}$ que contenían medio SOF con pronasa ( 5 $\mathrm{IU} / \mathrm{ml}$ ) (Sigma) (Sifer et al., 2006). Fueron transferidos a $50 \mu 1$ de medio SOF que contenía $5 \mu \mathrm{g} / \mathrm{ml}$ de Hoechst 33258 (Sigma) se incubaron a $37^{\circ} \mathrm{C}$ durante 8 minutos. Luego se adicionaron $5 \mu \mathrm{l}$ de ioduro de propidio (Sigma) para una concentración final de 100 $\mu \mathrm{g} / \mathrm{ml}$ y se incubó durante 8 minutos en condiciones de oscuridad. Posteriormente, se lavaron los embriones en $100 \mu 1$ de medio SOF y se evaluaron mediante la visualización en el microscopio de epifluorescencia E200 con HBO (Nikon Eclipse, EEUU), mediante un filtro UV-2A para las longitudes de onda entre 495/617 nm. Se realizó registro fotográfico de cada embrión y posteriormente se realizó el conteo de células vivas (azules) y muertas (rojas).

\section{Análisis Estadístico}

Se realizó un análisis de varianza, para el cual fueron evaluados previamente los supuestos de normalidad mediante la prueba 
Shapiro-Wilk y la homogeneidad de las varianzas mediante la prueba de Levene. La comparación de las medias se realizó mediante la prueba de Tukey. Todos los análisis se realizaron a través del programa SIGMA STAT (Systat Software, USA), considerando como significativo un valor $\mathrm{p}<0.05$.

\section{Resultados y Discusión}

Los valores encontrados para la producción de embriones bovinos in vitro (Cuadro 1) muestran un efecto de la quercetina dependiente de la concentración suplementada. Las dosis mayores afectaron las tasas de división y de blastocistos, aunque esto no se manifestó en el estadio de desarrollo de 4 o más células. Se ha reportado que las bajas dosis de quercetina $(2-10 \mu \mathrm{M})$ están asociadas con la protección contra las lesiones oxidantes, mientras que las altas dosis tienen un efecto opuesto (Johnson y Loo, 2000; Kang et al., 2013).

Aunque los flavonoides se caracterizan por presentar actividad antioxidante, bajo algunas circunstancias pueden formar un radi- cal aroxilo o de un complejo flavonoide hierro redox, resultando en una autooxidación del radical aroxilo, el cual produce un anión superóxido $\left(\mathrm{O}_{2}^{-}\right) \mathrm{y}$, este a su vez, genera el radical hidroxilo $\left(\mathrm{OH}^{-}\right)$, que puede tener efectos mutagénicos y citotóxicos (Hodnick et al., 1988; Hanasaki et al., 1994) inducidos por las altas concentraciones (Laughton et al., 1991; da Silva et al., 2002). Cao et al. (1997) revelaron que la quercetina en presencia de $\mathrm{Cu}^{2+}$ o $\mathrm{Fe}^{2+}$ libres actúa como prooxidante. Esto fue evidente en los resultados del presente estudio y consistente con lo hallado por Yu et al. (2014), quienes encontraron que la quercetina a $50 \mu \mathrm{M}$ disminuyó significativamente el desarrollo embrionario, actuando como un prooxidante. Entre tanto, Yu et al. (2014) y Sameni et al. (2018) afirman que la quercetina promueve el desarrollo de los embriones de ratón cuando se trabaja a $5 \mu \mathrm{M}$.

Cabe señalar que existen pocos reportes sobre el efecto de la quercetina en la producción in vitro de embriones bovinos; sin embargo, este antioxidante ha sido suplementado en la fase de maduración in vitro con éxito relativo (Gemra et al., 2013; Sovernigo et al., 2017). Así, Gemra et al. (2013) en-

Cuadro 1. Efecto de la quercetina sobre el cultivo de embriones bovinos producidos in vitro

\begin{tabular}{ccccc}
\hline $\begin{array}{c}\text { Quercetina } \\
(\mu \mathrm{M})\end{array}$ & $\begin{array}{c}\text { COC } \\
(\mathrm{n})\end{array}$ & $\begin{array}{c}\text { Clivaje } \\
\mathrm{n}(\%)\end{array}$ & $\begin{array}{c}>4 \text { células - Día 3 } \\
\mathrm{n}(\%)\end{array}$ & $\begin{array}{c}\text { Blastocistos - Día 7 } \\
\mathrm{n}(\%)\end{array}$ \\
\hline 1 & 255 & $219(85.9 \pm 0.09)^{\mathrm{a}}$ & $182(71.4 \pm 0.12)^{\mathrm{a}}$ & $105(41.2 \pm 0.10)^{\mathrm{ab}}$ \\
5 & 266 & $233(87.6 \pm 0.10)^{\mathrm{a}}$ & $212(79.7 \pm 0.13)^{\mathrm{a}}$ & $128(48.1 \pm 0.10)^{\mathrm{a}}$ \\
10 & 273 & $208(76.2 \pm 0.11)^{\mathrm{a}}$ & $176(64.5 \pm 0.15)^{\mathrm{a}}$ & $91(33.3 \pm 0.09)^{\mathrm{b}}$ \\
15 & 264 & $208(78.8 \pm 0.11)^{\mathrm{a}}$ & $166(62.9 \pm 0.24)^{\mathrm{a}}$ & $87(33.0 \pm 0.09)^{\mathrm{b}}$ \\
20 & 294 & $220(74.8 \pm 0.13)^{\mathrm{b}}$ & $203(69.0 \pm 0.12)^{\mathrm{a}}$ & $79(26.9 \pm 0.09)^{\mathrm{c}}$ \\
50 & 287 & $213(74.2 \pm 0.11)^{\mathrm{b}}$ & $188(65.5 \pm 0.11)^{\mathrm{a}}$ & $68(23.7 \pm 0.09)^{\mathrm{c}}$ \\
$\mathrm{C} 1$ & 244 & $204(83.6 \pm 0.06)^{\mathrm{a}}$ & $178(73.0 \pm 0.08)^{\mathrm{a}}$ & $92(37.7 \pm 0.07)^{\mathrm{b}}$ \\
$\mathrm{C} 2$ & 243 & $196(80.7 \pm 0.10)^{\mathrm{a}}$ & $165(67.9 \pm 0.12)^{\mathrm{a}}$ & $80(32.9 \pm 0.05)^{\mathrm{b}}$ \\
\hline
\end{tabular}

C1: tratamiento control con medio SOF y con $1 \mu \mathrm{l} / \mathrm{ml}$ de DMSO; 2 : Tratamiento control solo con medio SOF

Valores con diferentes superíndices (a-e) dentro de columnas denotan diferencias significativas $(p<0.05)$ 
Cuadro 2. Celularidad de blastocistos bovinos producidos in vitro en diferentes concentraciones de quercetina $(\mathrm{n}=30$ blastocistos por tratamiento)

\begin{tabular}{cl}
\hline Quercetina $(\mu \mathrm{M})$ & Células Totales \\
\hline 1 & $117.3 \pm 20.3^{\mathrm{a}}$ \\
5 & $133.5 \pm 37.3^{\mathrm{a}}$ \\
10 & $122.4 \pm 27.0^{\mathrm{a}}$ \\
15 & $111.2 \pm 27.0^{\mathrm{b}}$ \\
20 & $113.1 \pm 30.4^{\mathrm{a}}$ \\
50 & $113.5 \pm 25.2^{\mathrm{a}}$ \\
$\mathrm{C} 1$ & $129.6 \pm 23.9^{\mathrm{a}}$ \\
$\mathrm{C} 2$ & $124.5 \pm 28.1^{\mathrm{a}}$ \\
\hline
\end{tabular}

C1: tratamiento control con medio SOF y 1 $\mu \mathrm{l} / \mathrm{ml}$ de DMSO; C2: tratamiento control solo con medio SOF

Valores con diferentes superíndices denotan diferencias significativas $(p<0.05)$

contraron que la suplementación de los medios de maduración con quercetina $(2 \mu \mathrm{M})$ no mejoró las tasas de maduración nuclear ni de clivaje; mientras que Sovernigo et al. (2017) obtuvieron mayores tasas de producción de blastocistos y una reducción de EROs en los oocitos. La suplementación del medio de maduración con quercetina podría actuar como un mecanismo de defensa contra las EROs y podría contribuir a mantener el sistema glutatión (GSH) (Kovacic y Somanathan, 2010; Jeong y Joo, 2016). Sin embargo, no se ha reportado un incremento en los niveles de GSH utilizando quercetina en el medio de maduración (Gemra et al., 2013; Sovernigo et al., 2017). En cuanto al DMSO usado para disolver la quercetina, no se encontró un efecto significativo en las tasas de producción de blastocistos respecto al control con el medio SOF (Yu et al., 2014; Banihosseini et al., 2018).

Banihosseini et al. (2018) encontraron que las bajas concentraciones de quercetina en los medios de maduración in vitro aumentaron la formación de blastocistos de ratón; de forma similar a lo reportado por Kang et al. (2013) en porcinos. Así mismo, Guemra et al. (2013), informaron un incremento en las tasas de desarrollo cuando los oocitos bovinos fueron cultivados en medios con presencia de quercetina a bajas concentraciones $(2-10 \mu \mathrm{M})$, mientras que una alta concentración $(50 \mu \mathrm{M})$ causó un efecto deletéreo. Lo anterior ha sido asociado a su efecto prooxidante $o$ antioxidante, el cual es dependiente de la concentración (Yu et al., 2014). Se ha reportado que las altas concentraciones de EROs pueden generar estrés oxidativo que resulta en la inducción de la apoptosis en varios tipos de células (Bayir et al., 2008); sin embargo, los oocitos que son tratados con quercetina a bajas concentraciones presentan una reducción en los niveles de EROs (Banihosseini et al., 2018; Wang et al., 2017).

Kang et al. (2013) y Banihosseini et al. (2018) no hallaron diferencias en el número de células totales de los blastocistos porcinos producidos in vitro con quercetina, lo cual es similar a los resultados encontrados en esta investigación, donde solo se observó un efecto de la concentración de $15 \mu \mathrm{M}$ sobre la celularidad y no se observó dicho efecto a concentraciones superiores $(20$ y $50 \mu \mathrm{M})$ (Cuadro 2). Esto muestra que la quercetina es benéfica para la formación de blastocistos e interfiere poco en la celularidad de estos. A diferencia de este resultado, otros antioxidantes como el resveratrol (Lee et al., 2010) y la melatonina (Liang et al., 2017) han mostrado un efecto positivo sobre la celularidad.

Se ha demostrado que la quercetina puede inducir selectivamente la apoptosis en células cancerosas y evitarla en células normales (Liang et al., 2011; Park y Min do, 2011). Esto se ha probado tanto en fetos (Wang et al., 2016) como en blastocistos de ratón producidos in vitro, generando un mayor número de células viables ( $\mathrm{Yu}$ et al., 2014). Por su parte, Sameni et al. (2018) afirman que la quercetina promueve el desarrollo de los embriones de ratón y reduce la apoptosis en las blastómeras cuando se tra- 


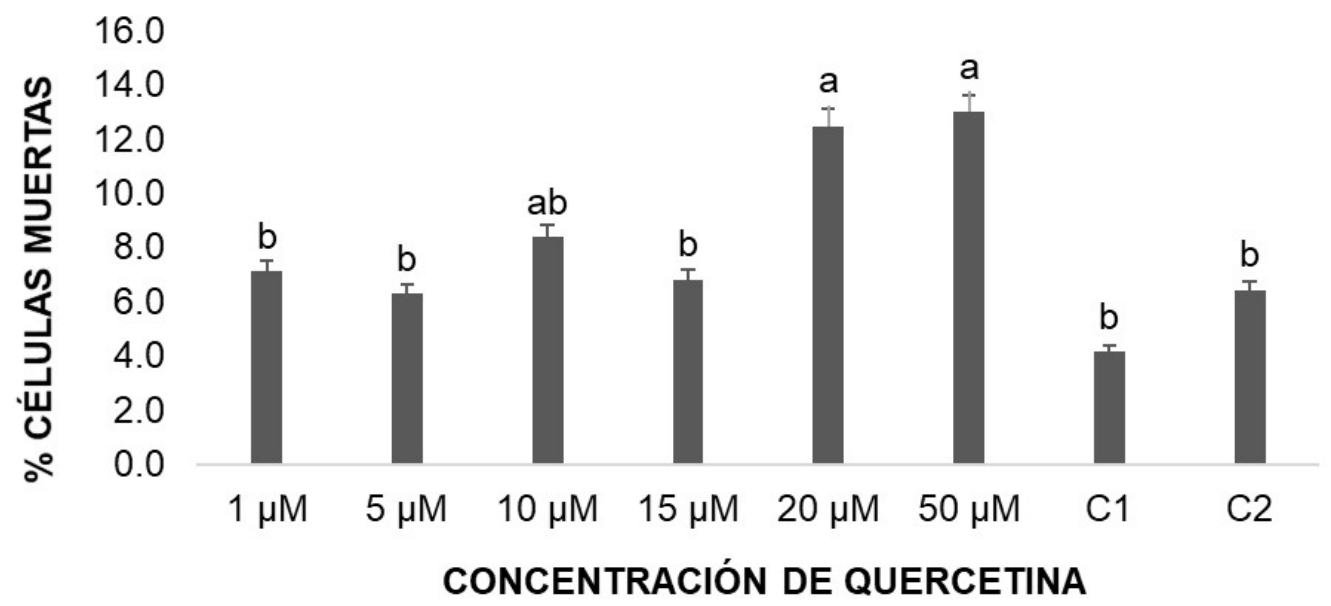

Figura 1. Viabilidad celular en blastocistos expandidos (día 7 de desarrollo). C1: tratamiento control con medio SOF y $1 \mu \mathrm{l} / \mathrm{ml}$ de DMSO; C2: tratamiento control solo con medio $\mathrm{SOF}$ (medias $\pm \mathrm{DE})$. Letras diferentes dentro de cada parámetro representan significancia estadística $(\mathrm{p}<0.05)$

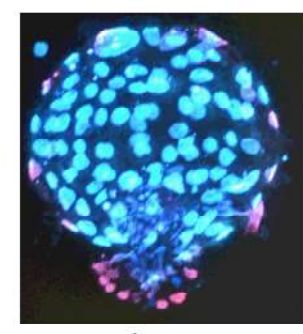

1

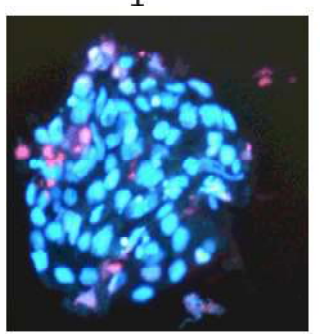

20

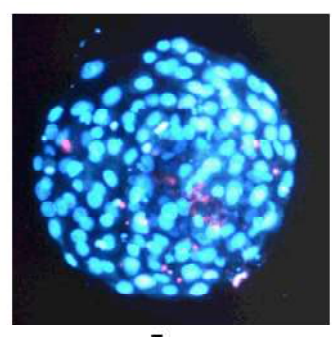

5

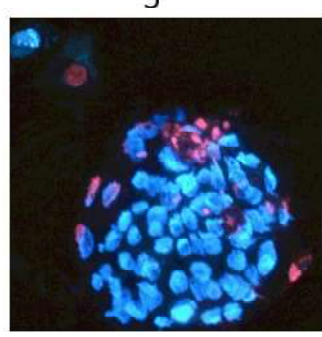

50

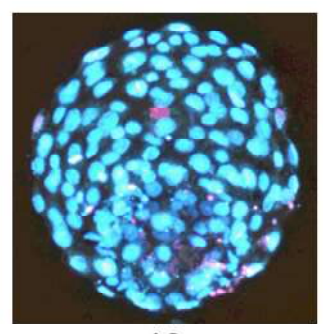

10

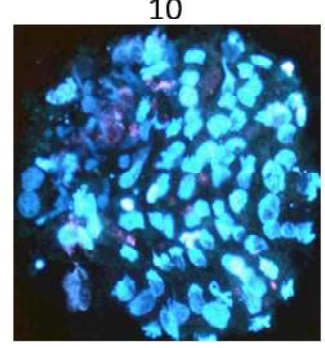

DMSO

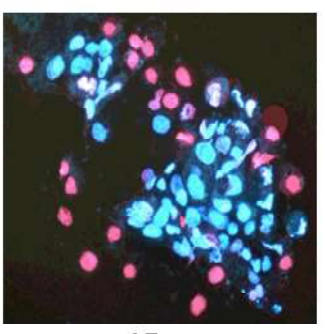

15

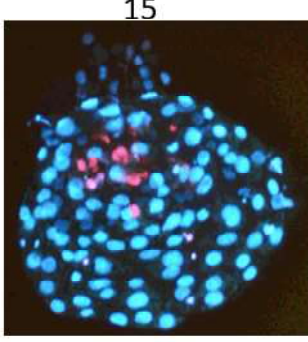

SOF

Figura 2. Células vivas y muertas de embriones bovinos producidos con diferentes concentraciones de quercetina 
baja a $5 \mu \mathrm{M}$. Lo anterior concuerda con los resultados del presente estudio, donde a bajas concentraciones no hay alteraciones en el número de células viables comparadas con el control, mientras que altas concentraciones resultan en un incremento de células muertas (Figuras 1 y 2), habiéndose sugerido que se debe a su efecto antioxidante (Heim et al., 2002). El mecanismo antioxidante de la quercetina se debe a su estructura polifenólica que son bien conocidos por su capacidad de interactuar con las EROs y ERNs mediante la transferencia de electrones (SET) y la transferencia de un átomo de hidrogeno (HAT); en el primer caso se cede un electrón al radical y en el segundo, el antioxidante cede un átomo de hidrógeno que estabiliza el radical (Zapata et al., 2014).

En el presente estudio se encontró que a altas concentraciones de quercetina se genera una reducción de la viabilidad celular en los blastocistos; a su vez, las bajas concentraciones no tienen efecto sobre la proporción de células muertas (Figura 1). En este sentido, otras investigaciones evidencian que la quercetina, al igual que otros antioxidantes, interfiere con la expresión de genes relacionados con la apoptosis y la calidad embrionaria (Bax, Blc-2, TP53, Oct-4, Cx43, IGF-1 y Glut-1 (Elamaran et al., 2012; You et al., 2012; Choi et al., 2013; Mukherjee et al., 2014; Kawashima et al., 2015). Es oportuno recalcar que todos los experimentos corroboran los efectos benéficos sobre la producción y calidad de embriones al suplementar la quercetina a bajas concentraciones en la fase de cultivo in vitro. Por lo tanto, sería importante determinar si la suplementación de quercetina durante todo el proceso de producción de embriones in vitro generaría mejores resultados.

\section{Conclusiones}

- La suplementación del medio de cultivo con quercetina a $1 \mu \mathrm{M}$ y $5 \mu \mathrm{M}$ genera un incremento en la producción de embriones bovinos in vitro.
- El uso de bajas concentraciones de quercetina en la fase de cultivo no interfiere con la celularidad y la viabilidad celular de los blastocistos bovinos producidos in vitro.

- El uso de altas concentraciones de quercetina en la fase de cultivo genera efectos deletéreos sobre la producción in vitro de embriones bovinos.

\section{Agradecimientos}

Al Politécnico Colombiano Jaime Isaza Cadavid y la Universidad Nacional de Colombia Sede Medellín, por la financiación de esta investigación.

\section{Literatura Citada}

1. Banihosseini SZ, Novin MG, Nazarian H, Piryaei A, Parvardeh S, Eini F. 2018. Quercetin improves develop-mental competence of mouse oocytes by reducing oxidative stress during in vitro maturation. Ann Anim Sci 18: 87-98. doi: 10.1515/aoas-2017-0029

2. Bayir H, Kagan VE. 2008. Bench-tobedside review: mitochondrial injury, oxidative stress and apoptosis-there is nothing more practical than a good theory. Crit Care 12: 206. doi: 10.1186/ cc6779

3. Caamaño JN, Gómez E, Trigal B, Muñoz M, Carrocera S, Martín D, Díez C. 2015. Survival of vitrified in vitroproduced bovine embryos after a onestep warming in-straw cryoprotectant dilution procedure. Theriogenology 83 : 881-890. doi: 10.1016/j.theriogenology.2014.11.021

4. Calegari RS, Crespilho AM, Martins $A$, Ferro AC. 2012. Produção de embriões bovinos in vitro em meio de cultura com ácido ascórbico. Vet Zootec 16: 80-88.

5. Cao G, Sofic E, Prior R. 1997. Antioxidant and prooxidant behaviour of flavonoids: structure-activity relationships. Free Radical Bio Med 22: 749-760. 
6. Choi JY, Kang JT, Park SJ, Kim SJ, Moon JH, Saadeldin IM, Jang G, et al. 2013. Effect of 7, 8-dihydroxyflavone as an antioxidant on in vitro maturation of oocytes and development of parthenogenetic embryos in pigs. $\mathrm{J}$ Reprod Develop 59: 450-456.

7. Coelho L, Esper C, Herrera R, Vantini R, Almeida I. 2002. Produção in vitro de embriões bovinos: utilização de diferentes fontes de gonadotrofinas na maturação dos oócitos. Rev Bras Zootecn 31: 1117-1121. doi: 10.1590/ S1516-35982002000500007

8. da Silva J, Herrmann SM, Peres W, Possa N, Gonzalez J, Erdtmann B. 2002. Evaluation of the genotoxic effect of rutin and quercetin by comet assay and micronucleus test. Food Chem Toxicol 40: 941-947. doi: 10.1016/S02786915(02)00015-7

9. Elamaran $G$, Singh $K$, Singh $M$, Singla S, Chauhan M, Manik R, Palta $P$. 2012. Oxygen concentration and cysteamine supplementation during in vitro production of buffalo (Bubalus bubalis) embryos affect mRNA expression of BCL-2, BCL-XL, MCL-1, BAX and BID. Reprod Domest Anim 47:10271036. doi: 10.1111/j.1439-0531.2012.02009.x

10. Geetha T, Malhotra V, Chopra K, Kaur IP. 2005. Antimutagenic and antioxidant/ prooxidant activity of quercetin. Indian J Exp Biol 43: 61-67.

11. Giaretta E, Spinaci M, Bucci D, Tamanini C, Galeati $G$ 2013. Effects of resveratrol on vitrified porcine oocytes. Oxid Med Cell Longev. doi: 10.1155/2013/920257

12. González R, Soto E, Delgado N, Ondiz A, Velarde J. 1992. Comparación de dos métodos de recolección de oocitos de ovarios de bovinos mestizos sacrificados. Rev Cient-Fac Cien V 11: 11-13.

13. Gordon MH, Roedig-Penman A. 1998. Antioxidant activity of quercetin and myricetin in liposomes. Chem Phys Lipids 97: 79-85. doi: 10.1016/S00093084(98)00098-X
14. Guemra, S, Monzani, PS, Santos ES, Zanin R, Ohashi OM, Miranda MS, et al. 2013. Maturação in vitro de oócitos bovinos em meios suplementados com quercetina e seu efeito sobre o desenvolvimento embrionário. Arq Bras Med Vet Zoo 65: 1616-1624. doi: 10.1590/S0102-09352013000600005

15. Guérin P, El Mouatassim S, Ménézo Y. 2001. Oxidative stress and protection against reactive oxygen species in the pre-implantation embryo and its surroundings. Hum Reprod Update 7: 175-189. doi: 10.1093/humupd/7.2.175

16. Hanasaki Y, Ogawa S, Fukui S. 1994. The correlation between active oxygens scavenging y antioxidant effects of flavonoids. Free Radical Bio Med 16: 845-850. doi: 10.1016/0891-5849(94)90202-X

17. Heim KE, Tagliaferro AR, Bobilya DJ. 2002. Flavonoid antioxidants: chemistry, metabolism and structureactivity relationships. J Nutr Biochem 13: 572-584. doi: 10.1016/S0955-2863(02)00208-5

18. Hodnick WF, Milosavljeviae EB, Nelson JH, Pardini RS. 1988. Electrochemistry of flavonoids. Biochem Pharmacol 37: 2607-2611.

19. Holm P, Booth PJ, Scsmidt MH Greve T, Callesen H. 1999. High bovine blastocyst development in a static in vitro production system using SOFaa medium supplemented with sodium citrate and myo-inositol with or without serumproteins. Theriogenology 52: 683-700. doi: 10.1016/S0093-691X(99)00162-4

20. Ishige K, Schubert D, Sagara Y. 2001. Flavonoids protect neuronal cells from oxidative stress by three distinct mechanisms. Free Radic Biol Med 30: 433-446. doi: 10.1016/S0891-5849(00)00498-6

21. Jeong CH, Joo SH. 2016. Downregulation of reactive oxygen species in apoptosis. J Cancer Prev 21: 13-20. doi: 10.15430/JCP.2016.21.1.13 
22. Johnson MK, Loo G. 2000. Effects of epigallocatechin gallate and quercetin on oxidative damage to cellular DNA. Mutat Res 459: 211-218. doi: 10.1016/S09218777(99)00074-9

23. Kang JT, Kwon DK, Park SJ, Kim SJ, Moon JH, Koo OJ, Jang G, Lee BC. 2013. Quercetin improves the in vitro development of porcine oocytes by decreasing reactive oxygen species levels. J Vet Sci. 14: 15-20. doi: 10.4142/ jvs.2013.14.1.15

24. Kang JT, Moon JH, Choi JY, Park SJ, Kim SJ, Saadeldin IM, Lee BC. 2016. Effect of antioxidant flavonoids (quercetin and taxifolin) on in vitro maturation of porcine oocytes. Asian Austral J Anim 29:352-358. doi: 10.5713/ ajas. 15.0341

25. Kawashima A, Sekizawa A, Koide K, Hasegawa J, Satoh K, Arakaki T, Takenaka S, et al. 2015. Vitamin C induces the reduction of oxidative stress and paradoxically stimulates the apoptotic gene expression in extravillous trophoblasts derived from first-trimester tissue. Reprod Sci 22: 783-790. doi: 10.1177/1933719114561561

26. Kordowitzki P, Bernal SM, Herrmann D, Aldag P, Niemann H. 2016. Resveratrol supplementation during in vitro maturation and fertilisation enhances developmental competence of bovine oocytes. Reprod Fert Develop 28: 230-230. doi: 10.1071/RDv28n2Ab198

27. Kovacic P, Somanathan, R. 2010. Multifaceted approach to resveratrol bioactivity: focus on antioxidant action, cell signaling and safety. Oxid Med Cell Longev 3: 86-100. doi: 10.4161/ oxim.3.2.11147

28. Kuwayama M, Leibo SP. 2008. Efficiency of the cryotop method to cryopreserve human oocytes analysis of in vitro and in vivo results at eleven IVF clinics. Int J Fertil Steril 90: 281-282. doi: 10.1016/j.fertnstert.2008.07.1089

29. Kwak SS, Cheong SA, Jeon Y, Lee E, Choi KC, Jeung EB, Hyun SH. 2012. The effects of resveratrol on porcine oocyte in vitro maturation and subsequent embryonic development after parthenogenetic activation and in vitro fertilization. Theriogenology 78: 86-101. doi: 10.1016/j.theriogenology.2012.01.024

30. Laughton MJ, Evans PJ, Moroney MA, Hoult JR, Halliwell B. 1991. Inhibition of mammalian 5-lipoxygenase and cyclo-oxygenase by flavonoids and phenolic dietary additives. Relationship to antioxidant activity and to iron ionreducing ability. Biochem Pharmacol 42: 1673-1681. doi: 10.1016/00062952(91)90501-U

31. Lee K, Wang C, Chaille J, Machaty Z. 2010. Effect of resveratrol on the development of porcine embryos produced in vitro. J Reprod Develop 56: 330-335. doi: 10.1262/jrd.09-174K

32. Leibfried L, First NL. 1979. Characterization of bovine follicular oocytes and their ability to mature in vitro. J Anim Sci 48: 76-86. doi: 10.2527/jas1979.48176x

33. Li M, Kildegaard KR, Chen Y, Rodriguez, A, Borodina I, Nielsen J. 2015. De novo production of resveratrol from glucose or ethanol by engineered Saccharomyces cerevisiae. Metab Eng 32: 1-11. doi: 10.1016/j.ymben.2015.08.007

34. Liang W, Li X, Li C, Liao L, Gao B, Gan H, Yang Z, Liao L, Chen X. 2011. Quercetin-mediated apoptosis via activation of the mitochondrial-dependent pathway in MG-63 osteosarcoma cells. Mol Med Report 4: 1017-1023. doi: 10.3892/mmr.2011.533.

35. Liang S, Jin YX, Yuan B, Zhang JB, Kim NH. 2017. Melatonin enhances the developmental competence of porcine somatic cell nuclear transfer embryos by preventing DNA damage induced by oxidative stress. Sci Rep 7: 11114. doi: 10.1038/s41598-017-11161-9. doi: 10.1038/s41598-017-11161-9.

36. Liu S, Hou W, Yao P, Zhang B, Sun S, Nüssler AK, Liu L. 2010. Quercetin protects against ethanol-induced oxidative damage in rat primary 
hepatocytes. Toxicol in vitro 24: 516522. doi: 10.1016/j.tiv.2009.03.006

37. Machado HK, Wanderley LS, Faustino LR, Da Silva CMG, De Figueiredo JR, Rodrigues APR. 2011. Papel de agentes antioxidantes na criopreservação de células germinativas e embriões. Acta Sci Vet 39: 956.

38. Merton JS, Knijn HM, Flapper H, Dotinga F, Roelen BAJ, Vos PLAM, Mullaart E. 2013. Cysteamine supplementation during in vitro maturation of slaughterhouse- and opu-derived bovine oocytes improves embryonic development without affecting cryotolerance, pregnancy rate, and calf characteristics. Theriogenology 80: 365-371. doi: 10.1016/j.theriogenology.2013.04.025

39. Moraes G, Silva S, Dos Santos D, Pasin M, Pereira L, Lourdes $M$, Mezzalira A, et al. 2002. Produção in vitro de embriões bovinos com soro de égua em diferentes fases do estro. Acta Sci Vet 30: 1-8.

40. Mukherjee A, Malik H, Saha AP, Dubey A, Singhal DK, Boateng $S$, Saugandhika S, et al. 2014. Resveratrol treatment during goat oocytes maturation enhances developmental competence of parthenogenetic and hand-made cloned blastocysts by modulating intracellular glutathione level and embryonic gene expression. J Assist Reprod Gen 31: 229-239. doi: 10.1007/ s10815-013-0116-9

41. Olson SE, Seidel GE. 2000. Culture of in vitro produced bovine embryos with vitamin E improves development in vitro and after transfer to recipients. Biol Reprod 62: 248-252. doi: 10.1095/ biolreprod62.2.248

42. Park MH, Min do S. 2011. Quercetininduced downregulation of phospholipase D1 inhibits proliferation and invasion in U87 glioma cells. Biochem Bioph Res Co 412: 710-715. doi: 10.1016/ j.bbrc. 2011.08 .037

43. Parrish J. 2014. Bovine in vitro fertilization: in vitro oocyte maturation and sperm capacitation with heparin.
Theriogenology 81: 67-73. doi: 10.1016/ j.theriogenology.2013.08.005

44. Phongnimitr T, Liang Y, Srirattana K, Panyawai K, Sripunya N, Treetampinich C, Parnpai R. 2013. Effect of L-carnitine on maturation, cryo-tolerance and embryo developmental competence of bovine oocytes. Anim Sci J 84: 719725. doi: $10.1111 /$ asj. 12067

45. Rahim B, Jalal S, Yosef N. 2011. Effect of cysteine supplementation on in vitro maturation of bovine oocyte. Afr. J. Biotechnol 10: 15830-15833. doi: 10.5897/AJB11.1686

46. Salzano A, Albero G, Zullo G, Neglia G, Abdel-Wahab A, Bifulco G, et al. 2014. Effect of resveratrol supplementation during culture on the quality and cryotolerance of bovine in vitro produced embryos. Anim Reprod Sci 151: 91-96. doi: 10.1016/ j.anireprosci.2014.09.018

47. Sameni HR, Javadinia SS, Safari M, Tabrizi MH, Khanmohammadi N, Parsaie H, Zarbakhsh S. 2018. Effect of quercetin on the number of blastomeres, zona pellucida thickness, and hatching rate of mouse embryos exposed to actinomycin D: an experimental study. Int J Reprod Biomed 16: 101-108.

48. Sifer C, Sellami A, Poncelet C, Kulski P, Martin-Pont B, Bottero J, Porcher $R$, et al. 2006. A prospective randomized study to assess the benefit of partial zona pellucida digestion before frozen-thawed embryo transfers. Hum Reprod 21: 2384-2389. doi: 10.1093/ humrep/del149

49. Sovernigo TC, Adona PR, Monzani PS, Guemra S, Barros F, Lopes FG, Leal C. 2017. Effects of supplementation of medium with different antioxidants during in vitro maturation of bovine oocytes on subsequent embryo production. Reprod 
Domest Anim 52: 561-569. doi: 10.1111/ rda.12946

50. Torres V, Urrego R, Echeverry JJ, Lopez A. 2016. 181 Resveratrol during in vitro maturation improves the quality of bovine oocyte and enhances embryonic. Reprod Fert Develop 29: 199-199. doi: 10.1071/RDv29n1 Ab181

51. Truong T, Gardner DK. 2017. Antioxidants improve IVF outcome and subsequent embryo development in the mouse. Hum Reprod 32: 2404-2413. doi: 10.1093/humrep/dex330

52. Wang F, Tian X, Zhang L, He C, Ji P, Li Y, Tan D, Liu G. 2014. Beneficial effect of resveratrol on bovine oocyte maturation and subsequent embryonic development after in vitro fertilization. Fertil Steril 101: 577-586. doi: 10.1016/ j.fertnstert.2013.10.041

53. Wang H, Jo YJ, Oh JS, Kim NH. 2017. Quercetin delays postovulatory aging of mouse oocytes by regulating SIRT expression and MPF activity. Oncotarget 8: 38631-38641. doi: 10.18632/oncotarget.16219

54. Wang $X$, Yan Y, Yang L, Li M, Zhong $X$. 2016. Effect of quercetin on the expression of $\mathrm{Bcl}-2 / \mathrm{Bax}$ apoptotic proteins in endometrial cells of lipopolysaccharide-induced-abortion mice. J Tradit Chin Med 36: 737-742.

55. You J, Lee J, Hyun SH, Lee E. 2012. L-carnitine treatment during oocyte maturation improves in vitro development of cloned pig embryos by influencing intracellular glutathione synthesis and embryonic gene expression. Theriogenology 78: 235-243. doi: 10.1016/j.theriogenology.2012.02.027

56. Yu S, Long H, Lyu QF, Zhang $Q H$, Yan ZG, Liang HX, Chai WR, Yan Z, Kuang YP, Qi C. 2014. Protective effect of quercetin on the development of preimplantation mouse embryos against hydrogen peroxide-induced oxidative injury. Plos One 9: e89520. doi: 10.1371/journal.pone.0089520

57. Zapata S, Piedrahita AM, Rojano B. 2014. Capacidad atrapadora de radicales oxígeno (ORAC) y fenoles totales de frutas y hortalizas de Colombia. Perspect Nutr Hum 16: 25-36.

58. Zullo G, Albero G, Neglia G, De Canditiis C, Bifulco G, Campanile $G$, Gasparrini B. 2016. L-ergothioneine supplementation during culture improves quality of bovine in vitro-produced embryos. Theriogenology 85: 688697. doi: 10.1016/j.theriogenology.-2015.-10.008 